\title{
Factors Influencing HIV Drug Resistance among Pregnant Women in Luanda, Angola: Findings from a Cross-Sectional Study
}

\author{
Cruz S. Sebastião ${ }^{1,2,3} \mathbb{C}^{\text {, Joana Morais }}{ }^{1,2,4}$ and Miguel Brito $1,5, * \mathbb{C}$ \\ 1 Centro de Investigação em Saúde de Angola, Caxito, Angola; cruzdossantos10@gmail.com (C.S.S.); \\ jmafonso.7@gmail.com (J.M.) \\ 2 Molecular Biology Laboratory, Instituto Nacional de Investigação em Saúde, Luanda, Angola \\ 3 Instituto Superior de Ciências da Saúde, Universidade Agostinho Neto, Luanda, Angola \\ 4 Faculdade de Medicina, Universidade Agostinho Neto, Luanda, Angola \\ 5 Health and Technology Research Center, Escola Superior de Tecnologia da Saúde de Lisboa, \\ Instituto Politécnico de Lisboa, 1990-096 Lisboa, Portugal \\ * Correspondence: miguel.brito@estesl.ipl.pt
}

Citation: Sebastião, C.S.; Morais, J.; Brito, M. Factors Influencing HIV Drug Resistance among Pregnant Women in Luanda, Angola: Findings from a Cross-Sectional Study. Trop.

Med. Infect. Dis. 2021, 6, 29

https://doi.org/10.3390/

tropicalmed6010029

Received: 4 February 2021

Accepted: 2 March 2021

Published: 5 March 2021

Publisher's Note: MDPI stays neutral with regard to jurisdictional claims in published maps and institutional affiliations.

Copyright: (c) 2021 by the authors. Licensee MDPI, Basel, Switzerland. This article is an open access article distributed under the terms and conditions of the Creative Commons Attribution (CC BY) license (https:// creativecommons.org/licenses/by/ $4.0 /)$.

\begin{abstract}
The increase in HIV infection and drug-resistant strains is an important public health concern, especially in resource-limited settings. However, the identification of factors related to the propagation of infectious diseases represents a crucial target offering an opportunity to reduce health care costs as well as deepening the focus on preventing infection in high-risk groups. In this study, we investigate the factors related to drug resistance among HIV-infected pregnant women in Luanda, the capital city of Angola. This was a part of a cross-sectional study conducted with 42 HIV-positive pregnant women. A blood sample was collected, and HIV-1 genotyping was carried out using an in-house method. Multivariate analyses were performed to determine the interaction between sociodemographic characteristics and drug resistance. HIV drug resistance was detected in $44.1 \%$ of the studied population. High probabilities of drug resistance were observed for HIV-infected pregnant women living in rural areas (AOR: 2.73; 95\% CI: 0.50-14.9) with high educational level (AOR: 6.27; 95\% CI: 0.77-51.2) and comorbidities (AOR: 5.47; 95\% CI: 0.28-106) and infected with a HIV-1 non-B subtype other than subtype C (AOR: 1.60; 95\% CI: 0.25-10.3). The present study reports high HIV drug resistance. Furthermore, older-age, rural areas, high educational levels, unemployed status, having comorbidities, and HIV-1 subtypes were factors related to drug resistance. These factors impact on drug susceptibility and need to be urgently addressed in order to promote health education campaigns able to prevent the spread of drug-resistant HIV strains in Angola.
\end{abstract}

Keywords: HIV infection; antiretroviral failure; risk factors; pregnant women; Angola

\section{Introduction}

The human immunodeficiency virus (HIV) remains a global public health problem [1] In 2019, an estimated 37.9 million people were living with HIV worldwide [2]. Of these, around 220,000 of the infected were living in Angola [2]. The HIV infection is caused by two lentiviruses (HIV-1 and HIV-2) divided into groups (M-P), subtypes (A-D, F-H, J, and $\mathrm{K}$ ), sub-subtypes (A1, A2, F1, and F2), and circulating recombinant forms (CRFs) [3].

The antiretroviral drugs currently approved to treat HIV infection belong to distinctive classes that act in different phases of the HIV replication as fusion inhibitor, nucleoside reverse transcriptase inhibitors (NRTIs), non-nucleoside reverse transcriptase inhibitors (NNRTIs), integrase inhibitors, and protease inhibitors (PIs) [4].

Countless factors, including drug-resistant strains, HIV subtypes, viral load monitoring, treatment adherence, comorbidities, and sociodemographic characteristics, have been associated with the development of drug resistance in HIV-infected pregnant women from low- and middle-income countries (LMICs) [5-10]. 
The identification of factors related to HIV drug resistance (HIVDR) represents a crucial goal to ensure the effectiveness of antiretroviral treatment (ART). To the best of our knowledge, this is the first study that investigates factors related to the spread of drug resistance mutation (DRMs) in HIV-positive pregnant women in Luanda, the capital of Angola. The information set out in this paper is an extension of our recently published study on HIV-1 diversity and drug resistance in pregnant women [11]. Hence, this study makes it possible to promote health education campaigns and strengthen the ongoing strategies in the control of HIVDR in Angola.

\section{Materials and Methods}

\subsection{Study Design and Setting}

This was part of a cross-sectional study carried out by the same research team with 42 HIV-infected pregnant women, recently diagnosed and without prior exposure to ART, between April and June 2018, at the Lucrecia Paim Maternity Hospital in Luanda, the capital of Angola. This is the largest public maternity unit and a reference center for maternal health care, training, and research in Angola. Furthermore, it is a tertiary health institution which provides health care for pregnant women and newborns from all national provinces. The main study inclusion criteria for pregnant women were confirmed diagnosis of HIV infection and no ART exposure. The study protocol was reviewed and approved by the Angolan National Ethics Committee (nr.13/2018) and the General Directorate of Lucrecia Paim Maternity, Luanda, Angola (nr.083/GDG/MLP/2018). The study was verbally explained to the participants and with written informed consent obtained from all participants, or the parents/legal guardians of minors aged under 15, before enrolment in the study.

\subsection{Data Collection and Procedure}

The research team collaborated with the hospital's teams for data collection. A structured questionnaire served to obtain sociodemographic characteristics, age, place of residence, level of education, occupation, and presence of comorbidities (Supplementary Material). For each participant, a blood sample was collected using vacutainers, and all prepared serum samples were stored at $-80^{\circ} \mathrm{C}$ until usage. HIV-1 genotyping test was carried out according to an in-house method as previously described [11]. The HIV nucleotide sequences obtained were submitted to the GenBank (NCBI) database and assigned accession numbers MK543512 to MK543545. HIV subtypes were confirmed by the REGA HIV-1 Subtyping Tool [12]. The identification of HIVDR was assessed with the genotypic resistance interpretation algorithm implemented in the Stanford HIV drug resistance database website (https:/ / hivdb.stanford.edu/hivdb/by-sequences/ accessed on 3 September 2018) [13].

\subsection{Statistical Analysis}

Statistical analyses were performed by SPSS v25 (IBM SPSS Statistics, Chicago, IL, USA). Categorical variables are presented as frequencies and percentages, and continuous variables as means and standard deviations (SD). Factors related to HIVDR were assessed using a logistic regression model. Univariate and multivariate analysis was performed with all independent variables and an outcome variable. All independent variables were included in the multivariate analysis to ensure that all potentially important variables were maintained. The goodness of fit for the model was verified by the Hosmer-Lemeshow test. The strength and direction of the relationship were determined with an adjusted odds ratio (AOR) and their 95\% confidence interval (CI). All reported $p$-values are two-tailed with a level of significance set at $5 \%$.

\section{Results}

All 42 pregnant women consented to participate in the study, provided the demographic data, and a blood sample for laboratory tests. Analysis of the HIV subtypes and 
DRMs could only be obtained in 34 out of the 42 samples subjected to HIV-1 genotyping. This was not possible in the remaining samples even after countless repeated attempts applying different conditions and PCR primers. Thus, only data on the 34 pregnant women successfully amplified and genotyped were included in the analyses.

Of the 34 samples genotyped, 15/34 (44.1\%) presented DRMs. Out of these 15 pregnant women with mutations, $14 / 15(93.3 \%), 2 / 15(13.3 \%)$, and $1 / 15(6.7 \%)$ presented resistance against the NNRTIs, NRTIs, and PIs, respectively. Furthermore, 2/15 (13.3\%) samples reported multidrug-resistance, one to NRTIs/NNRTIs and the other to PIs/NNRTIs. The mutations E44ED, M41L, D67N, T69D, and T215S were detected in the NRTIs, whereas the mutations V179E, E138A, V108I, V106I, G190A, P225H, K103N, Y181I, K103Q, and E138G were detected in the NNRTIs. The mutation L33F was the only one detected in the PIs (Table 1).

Table 1. Drug resistance mutations to nucleoside reverse transcriptase inhibitors (NRTIs), nonnucleoside reverse transcriptase inhibitors (NNRTIs), and protease inhibitors (PIs) according to the HIV-1 subtypes.

\begin{tabular}{lllc}
\hline \multirow{2}{*}{ HIV-1 Subtypes } & \multicolumn{3}{c}{ HIV-1 Drug Resistance Mutation } \\
\cline { 2 - 4 } & NRTI & NNRTI & PI \\
\hline F1 & - & V179E & - \\
F1/C & - & E138A & - \\
C & - & V108I & - \\
A1/G & - & V106I; G190A & - \\
F1 & - & P225H; K103N; & - \\
C & - & E138A & - \\
C & - & E138A & - \\
D & E44ED & E138A & - \\
H & - & - & - \\
G & M41L; D67N; T69D; & G190A & - \\
C & T215S & Y181I & - \\
CRF37_cpx & - & E138A & - \\
A1 & - & E138A & L33F \\
A1 & - & K103Q & - \\
C & - & E138G & - \\
\hline
\end{tabular}

All pregnant women were infected with the non-B subtype (nBS) from HIV-1 group M. Of these, subtype $C(n=13,38.2 \%)$ was the most frequent. Furthermore, subtypes F1, A1, G, D, H, F1/C, A1/G, H/G, CRF02_AG, and CRF37_cpx were found.

The putative factors related to HIVDR are set out in Table 2. The age range was 19 to 42 years. The mean age was $29 \pm 6$. Most of the pregnant women $(n=28,82.4 \%)$ were aged over 24 years, with high educational levels $(n=21,61.8 \%)$, employed $(n=19,55.9 \%)$, without comorbidities $(n=29,87.9 \%)$, and infected with HIV-1 nBS other than subtype C $(n=21,61.8 \%)$. The analysis returned no significant relationship $(p>0.05)$ and, even so, the multivariate analytical findings demonstrate that the likelihood of developing resistance in pregnant women from rural areas was 2.7 times (95\% CI: 0.50-14.9), with a high educational level was 6.3 times (95\% CI: 0.77-51.2), with comorbidities was 5.5 times (95\% CI: 0.28 106), and with HIV-1 nBS other than subtype C was 1.6 times (95\% CI: $0.25-10.3)$. On the other hand, pregnant women aged under 25 (AOR: 0.28 (95\% CI: 0.03-2.91), $p=0.287$ ) and employed pregnant women (AOR: 0.32 (95\% CI: $0.05-2.10), p=0.235$ ) were potential protective factors (Table 2). 
Table 2. Putative factors related to drug resistance among HIV-positive pregnant women in Luanda, Angola, 2018.

\begin{tabular}{|c|c|c|c|c|c|c|c|c|c|c|c|}
\hline \multirow{2}{*}{ Characteristics } & \multirow{2}{*}{$n(\%)$} & \multicolumn{2}{|c|}{ Resistance to NNRTIs } & \multicolumn{2}{|c|}{ Resistance to NRTIs } & \multicolumn{2}{|c|}{ Resistance to PIs } & \multicolumn{2}{|c|}{ Univariate Analysis } & \multicolumn{2}{|c|}{ Multivariate Analysis * } \\
\hline & & No $(\%)$ & Yes (\%) & No $(\%)$ & Yes $(\%)$ & No $(\%)$ & Yes $(\%)$ & OR $(95 \% \mathrm{CI})$ & $p$-Value & AOR $(95 \% \mathrm{CI})$ & $p$-Value \\
\hline $\begin{array}{c}\text { Overall } \\
\text { Age groups }\end{array}$ & $34(100)$ & $20(58.8)$ & $14(41.2)$ & $32(94.1)$ & $2(5.9)$ & $33(97.1)$ & $1(2.9)$ & & & & \\
\hline$<25$ years & $6(17.6)$ & $4(66.7)$ & $2(33.3)$ & $6(100)$ & $0(0.0)$ & $6(100)$ & $0(0.0)$ & $\begin{array}{c}0.58 \\
(0.09-3.68)\end{array}$ & 0.561 & $0.28(0.03-2.91)$ & 0.287 \\
\hline $\begin{array}{c}\geq 25 \text { years } \\
\text { Place of residence } b\end{array}$ & $28(82.4)$ & $16(57.1)$ & $12(42.9)$ & $26(92.9)$ & $2(7.1)$ & $27(96.4)$ & $1(3.6)$ & 1.00 & - & 1.00 & - \\
\hline Urban area & $17(50.0)$ & $12(70.6)$ & $5(29.4)$ & $16(94.1)$ & $1(5.9)$ & $16(94.1)$ & $1(5.9)$ & 1.00 & - & 1.00 & - \\
\hline Rural area & $17(50.0)$ & $8(47.1)$ & $9(52.9)$ & $16(94.1)$ & $1(5.9)$ & $17(100)$ & $0(0.0)$ & $\begin{array}{c}2.06 \\
(0.52-8.18)\end{array}$ & 0.303 & $2.73(0.50-14.9)$ & 0.247 \\
\hline $\begin{array}{c}\text { Education }^{\mathrm{c}} \\
\text { Low }\end{array}$ & $13(38.2)$ & $10(76.9)$ & $3(23.1)$ & $12(92.3)$ & $1(7.7)$ & $13(100)$ & $0(0.0)$ & 1.00 & - & 1.00 & - \\
\hline High & $21(61.8)$ & $10(47.6)$ & $11(52.4)$ & $20(95.2)$ & $1(4.8)$ & $20(95.2)$ & $1(4.8)$ & $\begin{array}{c}2.48 \\
(0.58-10.6)\end{array}$ & 0.223 & $6.27(0.77-51.2)$ & 0.086 \\
\hline $\begin{array}{l}\text { Occupation }^{\mathrm{d}} \\
\text { Unemployed }\end{array}$ & $15(44.1)$ & $8(53.3)$ & 7 (46.7) & $14(93.3)$ & $1(6.7)$ & $14(93.3)$ & $1(6.7)$ & 1.00 & - & 1.00 & - \\
\hline Employed & $19(55.9)$ & $12(63.2)$ & $7(36.8)$ & 18 (94.7) & $1(5.3)$ & $19(100)$ & $0(0.0)$ & $\begin{array}{c}0.51 \\
(0.13-2.02)\end{array}$ & 0.339 & $0.32(0.05-2.10)$ & 0.235 \\
\hline Yes & $4(12.1)$ & $1(25.0)$ & $3(75.0)$ & $4(100)$ & $0(0.0)$ & $4(100)$ & $0(0.0)$ & $\begin{array}{c}4.91 \\
(0.45-53.3)\end{array}$ & 0.191 & $5.47(0.28-106)$ & 0.261 \\
\hline $\begin{array}{l}\text { HIV-1 nBS } \\
\text { Subtype C }\end{array}$ & $13(38.2)$ & $8(61.5)$ & $5(38.5)$ & $13(100)$ & $0(0.0)$ & $13(100)$ & $0(0.0)$ & 1.00 & - & 1.00 & - \\
\hline Others nBS & $21(61.8)$ & $12(57.1)$ & $9(42.9)$ & 19 (90.5) & $2(9.5)$ & $20(95.2)$ & $1(4.8)$ & $\begin{array}{c}1.46 \\
(0.36-5.95)\end{array}$ & 0.602 & $1.60(0.25-10.3)$ & 0.619 \\
\hline
\end{tabular}

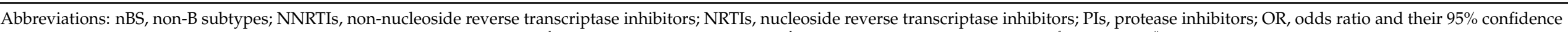

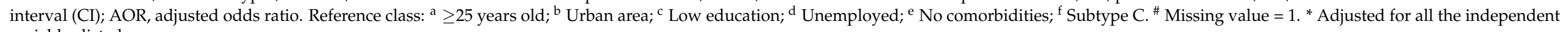
variables listed. 


\section{Discussion}

Access to ART is important for reducing mortality and prevents HIV transmission $[14,15]$. These public health accomplishments were made possible by the widespread administration of standardized ART regimens characterized by an inexpensive fixed-dose combination of NRTIs plus NNRTIs [16]. Although efforts are being made to control HIV, infected people can acquire DRM and can also be infected with drug-resistant strains [17].

In this study, we report a small study with ART-naïve pregnant women in terms of the burden of transmitted DRMs, and to investigate factors related to increasing HIVDR. We found a high $(\geq 15 \%)$ drug resistance level according to the WHO [18]. The resulting resistance level was thus higher than those reported in pregnant women from Ghana $(<5 \%)$ [5], Nigeria $(<5 \%)$ [7], Malawi $(7.6 \%)$ [8], and Guinea Bissau $(10.4 \%)$ [10], while lower than that returned in Tanzania (97\%) [9]. The drug resistance level observed in these pregnant women represents a threat to Angola's ART program $[19,20]$.

The identification of the sociodemographic factors of the HIV-affected population is a crucial target for intervention and control of the emergence of DRMs. To the best of our knowledge, this is the first study on factors related to the scatter of HIVDR among pregnant women from Angola. Age, patient residence, educational level, occupation, comorbidities, and HIV-1 subtypes were found to be factors related to the risk of ART failure supported by results recorded by previous studies in LMICs [5-10].

Consistent with our findings, Wilhelmson et al. [10] and Imade et al. [7] reported a high frequency of DRMs in pregnant women aged over 24 years in Guinea Bissau and Nigeria, respectively. Our results are also similar to the findings of Khienprasit et al. [21], which reported a lower likelihood of developing treatment failure in HIVpositive women from urbanized areas, whereas the HIV-positive pregnant women with comorbidities $(\mathrm{OR}=1.49(95 \% \mathrm{CI}=1.03-2.15), p=0.032)$ were associated with treatment failure. By contrast, Chagomerana et al. [8] identified how HIV-positive pregnant women with high educational levels were less likely to develop treatment failure, while Wilhelmson et al. observed unfavorable virologic outcomes in pregnant women with low educational level [10].

The protective factor observed in pregnant women aged under 25 years was consistent with studies performed by Khienprasit et al. [21] and Chao et al. [22]. By contrast, CrabtreeRamírez et al. [23] reported higher odds related to ART failure in young women (OR: 4.7, (95\% CI: 1.5-14.4), $p=0.003)$. On the other hand, the protective factor for employed pregnant women was consistent with a study carried out by Bayu et al. [24].

Based on these results, a focus on younger women, employed and from urbanized areas, should be prioritized to ensure better ART outcomes. Additionally, it is urgent to increase the screening for comorbidities and points of care to detect ART failure in rural areas.

Studies showed improved virological outcomes in patients with nBS [25]. However, a widely discussed issue is the impact of ART on nBS once drug design has been performed on subtype B and extended to nBS [26]. Herein, we detect a high likelihood of present DRM in women with other nBS compared to subtype C (AOR: 1.60) (Table 2). For this reason, an update of the algorithms to interpret HIVDR and assess the drugs to which their virus is susceptible should be considered prior to the selection of ART regimens [27].

Our study has limitations. The small sample size limits the conclusions of our study. Besides, this study does not represent the whole population of pregnant women from Luanda or other regions from Angola. The HIV viral load, CD4 count, and other clinical factors were not determined in the enrolled subjects due to a lack of laboratory resources. A low viral load or the high genetic diversity of HIV-1 subtypes circulating in Luanda may explain the failure of the amplification of the viral RNA of the remaining eight samples. Further studies with a larger sample size and participants from other communities in Angola are needed to elucidate the burden of HIVDR in Angolan women. Furthermore, in settings with a poor roll-out of virological load monitoring, the investigation of the factors 
related to drug resistance will generate a great impact on the development of more effective public health strategies to control the HIV epidemic in LMICs.

\section{Conclusions}

Our findings show that HIV drug resistance is a public health burden among pregnant women in Luanda that needs addressing. Older-aged, rural areas, a high educational level, unemployed, comorbidities, and HIV-1 non-B subtypes were factors related to the increase in drug resistance in pregnant women from Luanda. Therefore, if the risk factors related to the spread of HIV drug resistance are not urgently addressed, they may increase hard-to-treat infections and health care costs in Angola.

Supplementary Materials: The following are available online at https:/ www.mdpi.com/2414-636 6/6/1/29/s1.

Author Contributions: Study design and conceptualization: M.B., J.M., and C.S.S. Sample collection: C.S.S. Laboratory analysis: C.S.S. Data analysis: M.B., C.S.S. Writing of the manuscript: M.B. and C.S.S. Revision of the manuscript: All authors revised the manuscript, read and approved the final version. All authors have read and agreed to the published version of the manuscript.

Funding: This research was funded by Camões, Institute of Cooperation and Language, Portugal; Calouste Gulbenkian Foundation, Portugal; the government of Bengo Province and the Angolan Ministry of Health. CCS holds a grant from the Foundation for Science and Technology (FCT) (SFRH/BD/135296/2017). H\&TRC authors gratefully acknowledge FCT/MCTES national support through UIDB/05608/2020 and UIDP/05608/2020.

Institutional Review Board Statement: The study protocol was reviewed and approved by the Angolan National Ethics Committee (nr.13/2018), and the General Directorate of Lucrecia Paim Maternity (nr.083/GDG/MLP/2018).

Informed Consent Statement: Written informed consent was obtained from all subjects involved in the study.

Data Availability Statement: The datasets generated during and/or analysed during the current study are available from the corresponding author on reasonable request.

Acknowledgments: We are extremely grateful to all the study participants. We wish to thank the Postgraduate Science for Development Program (PGCD) and the Foundation for Science and Technology (FCT), for the Ph.D. scholarship awarded to CSS (grant number SFRH/BD/135296/2017). Thanks to participant investigators from ISCISA/UAN for their contribution to the collected data. Thanks to investigators of the molecular biology laboratory from INIS and IOC/FIOCRUZ for laboratory support. Thanks to clinical investigators from INLS and Lucrecia Paim Maternity for institutional support.

Conflicts of Interest: The authors declare no conflict of interest.

\section{References}

1. UNGASS. Political Declaration on HIV and AIDS: On the Fast-Track to Accelerate the Fight against HIV and to End the AIDS Epidemic by 2030; UNAIDS: Geneva, Switzerland, 2016; Volume 56350, pp. 1-27.

2. UNAIDS. UNAIDS UNAIDS Data 2019 Reference. Joint United Nations Programme on HIV/AIDS; UNAIDS: Geneva, Switzerland, 2019.

3. Foley, B.T.; Leitner, T.K.; Apetrei, C.; Hahn, B.; Mizrachi, I.; Mullins, J.; Rambaut, A.; Wolinsky, S.; Korber, B.T.M. HIV Sequence Compendium 2015; National Institutes of Health (NIH): Los Alamos, NM, USA, 2015.

4. World Health Organization (WHO). Consolidated Guidelines on the Use of Antiretroviral Drugs for Treating and Preventing HIV Infection. Recommendations for a Public Health Approach (2016), 2nd ed.; WHO: Geneva, Switzerland, 2017.

5. Bonney, E.Y.; Addo, N.A.; Ntim, N.A.A.; Addo-Yobo, F.; Bondzie, P.; Aryee, K.E.; Barnor, J.; Brandful, J.; Bekoe, V.; Ohene, S.A.; et al. Low level of transmitted HIV Drug resistance at two HIV care centres in Ghana: A threshold survey. Ghana Med. J. 2013, 47, 82-86. [PubMed]

6. Wallis, C.L.; Godfrey, C.; Fitzgibbon, J.E.; Mellors, J.W. Key Factors Influencing the Emergence of Human Immunodeficiency Virus Drug Resistance in Low- and Middle-Income Countries. J. Infect. Dis. 2017, 216, 851-856. [CrossRef] [PubMed] 
7. Imade, G.E.; Sagay, A.S.; Chaplin, B.; Chebu, P.; Musa, J.; Okpokwu, J.; Hamel, D.J.; Pam, I.C.; Agbaji, O.; Samuels, J.; et al. Short communication: Transmitted HIV drug resistance in antiretroviral-naive pregnant women in north central Nigeria. AIDS Res. Hum. Retrovir. 2014, 30, 127-133. [CrossRef] [PubMed]

8. Chagomerana, M.B.; Miller, W.C.; Tang, J.H.; Hoffman, I.F.; Harrington, B.J.; DiPrete, B.; Wallie, S.; Jumbe, A.; Limarzi, L.; Hosseinipour, M.C. Prevalence of antiretroviral therapy treatment failure among HIV-infected pregnant women at first antenatal care: PMTCT option b+ in Malawi. PLoS ONE 2018, 13, 1-12. [CrossRef] [PubMed]

9. Ngarina, M.; Kilewo, C.; Karlsson, K.; Aboud, S.; Karlsson, A.; Marrone, G.; Leyna, G.; Ekström, A.M.; Biberfeld, G. Virologic and immunologic failure, drug resistance and mortality during the first 24 months postpartum among HIV-infected women initiated on antiretroviral therapy for life in the Mitra plus Study, Dar es Salaam, Tanzania. BMC Infect. Dis. 2015, 15, 1-10. [CrossRef] [PubMed]

10. Wilhelmson, S.; Månsson, F.; Lindman, J.L.; Biai, A.; Esbjörnsson, J.; Norrgren, H.; Jansson, M.; Medstrand, P. Prevalence of HIV-1 pretreatment drug resistance among treatment naïve pregnant women in Bissau, Guinea Bissau. PLoS ONE 2018, 13, 1-12. [CrossRef] [PubMed]

11. Sebastião, C.S.; Neto, Z.; De Jesus, C.S.; Mirandela, M.; Jandondo, D.; Couto-Fernandez, J.C.; Tanuri, A.; Morais, J.; Brito, M. Genetic diversity and drug resistance of HIV-1 among infected pregnant women newly diagnosed in Luanda, Angola. PLoS ONE 2019, 14, e0225251. [CrossRef] [PubMed]

12. Pineda-Peña, A.-C.; Varanda, J.; Sousa, J.D.d.; Kristof Theys, I.B.; Leitner, T.; Taveira, N.; Vandamme, A.-M.; Abecasis, A.B. On the contribution of Angola to the initial spread of HIV-1 Andrea-Clemencia. Infect. Genet. Evol. 2018, 2016, $219-222$.

13. Liu, T.F.; Shafer, R.W. Web Resources for HIV Type 1 Genotypic-Resistance Test Interpretation. Clin. Infect. Dis. 2006, 42, 1608-1618. [CrossRef] [PubMed]

14. Rodger, A.J.; Cambiano, V.; Bruun, T.; Vernazza, P.; Collins, S.; Van Lunzen, J.; Corbelli, G.M.; Estrada, V.; Geretti, A.M.; Beloukas, A.; et al. Sexual Activity Without Condoms and Risk of HIV Transmission in Serodifferent Couples When the HIV-Positive Partner Is Using Suppressive Antiretroviral Therapy. JAMA 2016, 316, 171. [CrossRef] [PubMed]

15. Rodger, A.J.; Cambiano, V.; Phillips, A.N.; Bruun, T.; Raben, D.; Lundgren, J.; Vernazza, P.; Collins, S.; Degen, O.; Corbelli, G.M.; et al. Risk of HIV transmission through condomless sex in serodifferent gay couples with the HIV-positive partner taking suppressive antiretroviral therapy (PARTNER): Final results of a multicentre, prospective, observational study. Lancet 2019, 393, 2428-2438. [CrossRef]

16. World Health Organization (WHO). Antiretroviral Medicines in Low-and Middle-Income Countries; World Health Organization: Geneva, Switzerland, 2013.

17. World Health Organization (WHO). HIV Drug Resistance Report 2019; World Health Organization: Geneva, Switzerland, 2019; ISBN 9789241503938.

18. WHO. WHI WHO Global Strategy for the Surveillance and Monitoring of HIV Drug Resistance 2012; WHO: Geneva, Switzerland, 2014.

19. Instituto Nacional de Luta Contra a Sida. Plano Estratégico Nacional Para o Controlo das Infecções de Transmissão Sexual, VIH e Sida. Angola; Instituto Nacional de Luta Contra a Sida: Luanda, Angola, 2006.

20. Instituto Nacional de Luta Contra a Sida. Normas de Tratamento Antiretroviral, Angola; Instituto Nacional de Luta Contra a Sida: Luanda, Angola, 2015.

21. Khienprasit, N.; Chaiwarith, R.; Sirisanthana, T.; Supparatpinyo, K. Incidence and risk factors of antiretroviral treatment failure in treatment-naïve HIV-infected patients at Chiang Mai University Hospital, Thailand. AIDS Res. Ther. 2011, 8, 42. [CrossRef] [PubMed]

22. Chao, C.; Tang, B.; Hurley, L.; Silverberg, M.J.; Towner, W.; Preciado, M.; Horberg, M. Risk factors for short-term virologic outcomes among HIV-infected patients undergoing regimen switch of combination antiretroviral therapy. AIDS Res. Hum. Retrovir. 2012, 28, 1630-1636. [CrossRef] [PubMed]

23. Crabtree-Ramírez, B.; Villasís-Keever, A.; Galindo-Fraga, A.; Del Río, C.; Sierra-Madero, J. Effectiveness of highly active antiretroviral therapy (HAART) among HIV-infected patients in Mexico. AIDS Res. Hum. Retrovir. 2010, 26, 373-378. [CrossRef] [PubMed]

24. Bayu, B.; Tariku, A.; Bulti, A.B.; Habitu, Y.A.; Derso, T.; Teshome, D.F. Determinants of virological failure among patients on highly active antiretroviral therapy in University of Gondar Referral Hospital, Northwest Ethiopia: A case-control study. HIV/AIDS Res. Palliat. Care 2017, 9, 153-159. [CrossRef] [PubMed]

25. Scherrer, A.U.; Ledergerber, B.; Von Wyl, V.; Böni, J.; Yerly, S.; Klimkait, T.; Bürgisser, P.; Rauch, A.; Hirschel, B.; Cavassini, M.; et al. Improved virological outcome in white patients infected with HIV-1 non-B subtypes compared to subtype B. Clin. Infect. Dis. 2011, 53, 1143-1152. [CrossRef] [PubMed]

26. Vergne, L.; Snoeck, J.; Aghokeng, A.; Maes, B.; Valea, D.; Delaporte, E.; Vandamme, A.M.; Peeters, M.; Laethem, K. Van Genotypic drug resistance interpretation algorithms display high levels of discordance when applied to non-B strains from HIV-1 naive and treated patients. FEMS Immunol. Med. Microbiol. 2006, 46, 53-62. [CrossRef] [PubMed]

27. Wagner, S.; Kurz, M.; Klimkait, T. Algorithm evolution for drug resistance prediction: Comparison of systems for HIV-1 genotyping. Antivir. Ther. 2015, 20, 661-665. [CrossRef] [PubMed] 